\title{
Analysis, Design, Simulation and Evaluation of Sigma-Delta $(\Sigma \delta)$ Modulator for Gsm Synthesizer
}

\author{
Hadi T. Ziboon and Haider R. Karim \\ Department of Electrical and Electronic Engineer, UOT., Baghdad-Iraq.
}

\begin{abstract}
The analysis, design, simulation and evaluation of $2^{\text {nd }}, 3^{\text {rd }}$ and $4^{\text {th }}$ order $\Sigma \Delta$ modulator and loop filter respectively are discussed, in this paper, to show their impact on the performance of fractional-N PLL-FS for GSM system. All simulation results show that the system is stable. The resulting settling time, spurious level and phase noise at $20 \mathrm{MHz}$ offset frequency of this synthesizer for $2^{\text {nd }}, 3^{\text {rd }}$ and $4^{\text {th }}$ order $\Sigma \Delta$ modulator and loop filter respectively are $2.92 \mu \mathrm{s},-35 \mathrm{dBc}$, $-164 \mathrm{dBc} / \mathrm{Hz}, 3.28 \mu \mathrm{s},-64 \mathrm{dBc},-186 \mathrm{dBc} / \mathrm{Hz}, 3.38 \mu \mathrm{s},-79 \mathrm{dBc}$ and $-190 \mathrm{dBc} / \mathrm{Hz}$ for $2^{\text {nd }}, 3^{\text {rd }}$ and $4^{\text {th }}$ order respectively. These results show the improvement in the spurious level and phase noise by $-19 \mathrm{dBc},-31 \mathrm{dBc} / \mathrm{Hz}$ for $3^{\text {rd }}$ order system and $-34 \mathrm{dBc},-35 \mathrm{dBc} / \mathrm{Hz}$ for $4^{\text {th }}$ order system respectively compared to the published work. CppSim program and Matlab (R2007a) are used for the simulation of $\Sigma \Delta$ fractional-N PLL-FS.
\end{abstract}

Keywords: GSM, PLL, Loop filter, Sigma-Delta $(\Sigma \Delta)$, Frequency Synthesizer (FS).

\section{Introduction}

To constitute a complete transceiver for wireless communication systems, one indispensable building block, is required by both the receive and transmit path, is the Frequency Synthesizer (FS) to generate the frequency (or frequencies) signal. Wherever frequencies are translated, frequency synthesis is crucial to provide a clean, stable and programmable Local Oscillator (LO) signal. Programmable to address all frequency channels with fast switching and high resolution are required to perform the addressing sufficiently fast. The low noise level is vital for the quality and reliability of the information transfer [1].

Fractional-N Phase-Locked Loop (PLL)FS technique is the most advanced and recent technique to satisfy these requirements. Fractional-N is a technique to achieve frequency resolution finer than the reference frequency. Fractional-N synthesis can be divided into two categories, classical fractional-N synthesis and sigma-delta $(\Sigma \Delta)$ fractional-N synthesis. The main performance limitation of the classical approach to fractional-N synthesis centers around the difficulty in creating a precise match between the noise cancellation Digital-to-Analog Converter (DAC) output and the phase error signal [2]. In $\Sigma \Delta$ fractional-N synthesis, the most popular technique used today to generate fractional divide values, and the spurious performance is improved through $\Sigma \Delta$ modulation of the divider control. The quantization noise introduced by dithering the divide value is therefore whitened and shaped to high frequencies, such that it is substantially filtered by the synthesizer dynamics [3].

\section{GSM System}

The GSM system is a system with a wide spread use and the typical configuration for GSM system is shown in Fig. (1). The main blocks for GSM system cell are Mobile Stations (MS) communicating with a network of Base Transceiver Station (BTS). Each cell in the cellular network requires a Radio Frequency (RF) carrier. The original cell is divided into three smaller cells. These cells share the same cell site but each has its own allocation of radio carriers. An RF carrier is a pair of radio frequencies. One is used in each direction (including transmission and reception) so that information may be passed in both directions simultaneously [4]. The frequency spectrum allocated for cellular system is only a narrow bandwidth. The bandwidth for the GSM system is $25 \mathrm{MHz}$. The transmitting and receiving frequencies in GSM are separated by $45 \mathrm{MHz}$ to avoid interference. The frequency bandwidth used for the downlink (from BTS to MS) is 
(935-960) MHz. The frequency bandwidth for the uplink (from MS to BTS) is (890-915) MHz. The channel spacing is $200 \mathrm{KHz}$ [5]. Frequency synthesis is an essential technique employed in RF systems to achieve LO generation or direct modulation transmission. Radio Frequency (RF) system designers of Time Division Multiple Access (TDMA) based cellular systems, such as GSM, need LO or FS blocks capable of tuning to a new channel within a small fraction of each time slot. The suppression of reference spurs and phase noise is also critical for these modern digital standards. Base station and data transmission applications are now striving to utilize all the time slots available in each frame using a single synthesizer [6].

\section{Frequency Synthesizer Parameters}

Many different parameters must be considered to determine the cost, weight and power consumption of a synthesizer in a particular application. The most common parameters are described below $[6,7,8]$ :

-Frequency resolution: This parameter is also referred to as the step size, and it specifies the minimum step size of the frequency increments.

-Settling time: This parameter is defined as the maximum amount of time required for the output frequency to reach a stable state at which the FS can hop from one frequency to another.

-Spurious signals: The spurious signal is defined as the ratio of the spur (also known as tone) power at a certain frequency offset to the carrier power expressed in $\mathrm{dBc}$.

-Phase noise: It is the random fluctuations in the phase of output frequency of PLL [9]. The Single SideBand (SSB) phase noise is defined as the ratio of noise power in $1 \mathrm{~Hz}$ bandwidth at a certain frequency offset $(\Delta f)$ from the carrier (ime) to the carrier power ( $P_{\text {mantos }}$ ) expressed in $\mathrm{dBc} / \mathrm{Hz}[8,10]$. A low phase noise VCO is an essential building block for the synthesizer. The phase noise requirements by the GSM $900 \mathrm{MHz}$ standard system are shown below in Table (1) [11].

\section{System Layout}

The general layout of the system design is shown in Fig. (2). The design parameters for the $\Sigma \Delta$ fractional-N PLL for GSM synthesizer are shown in Table (2).

\section{Model of Quantization Noise for PFD/DAC}

It is desirable to noise-shape the cancellation DAC's quantization noise. This is accomplished by processing the residue of the divider control with a $\Sigma \Delta$ modulator [12], as shown in Fig. (3). In order to achieve frequency resolution, the input word length of the $\Sigma \Delta$ modulator is chosen as 20-bits [13, 14]. The resolution of the $\Sigma \Delta$ modulator [15], is given by:

$$
f_{\text {resolution }}=\frac{f_{\text {ref }}}{2^{E}}
$$

where $1:$ Number of bit input to the $\Sigma \Delta$ modulator.

frof Reference frequency.

The output of the $\Sigma \Delta$ modulator (divider control), [16] is given by:

$$
v(\gamma)-r(x)+r_{2} \cdot\left(-z^{-1}\right)
$$

where $E_{g}$ : Quantization noise of the $1^{\text {st }}$ order $\Sigma \Delta$ modulator.

$F(z):$ Input of the $1^{\text {st }}$ order $\Sigma \Delta$ modulator.

a: Number of bit input to the $\Sigma \Delta$ modulator.

The design parameters of the $1^{\text {st }}$ order $\Sigma \Delta$ modulator (divider control) are shown in Table (3 a). The quantization noise shaping transfer function for $\Sigma^{\text {th }}$ order MASH $\Sigma \Delta$ modulator, [12] is given by:

$$
N T F=\left(1-Z^{-1}\right)^{2} / \Delta
$$

where $\mathrm{A}$ is the quantization step size.

The quantization noise reduction produced by the PFD/DAC is accomplished by dividing the noise transfer function of $\Sigma \Delta$ modulator by $2^{5}$ and is given by [12]:

$$
N T V-\frac{\left(1-z^{-1}\right)}{2^{3}}
$$


where $\bar{\nabla}:$ Number of bit of the DAC .

More levels are required to properly cancel the quantization noise [12]. The Signal-toQuantization Noise Ratio (SQNR) achieved by a noise shaped PFD/DAC synthesizer, [17], is given by:

SQNR $=6.02 \Re \bar{B} \quad-76 \quad d E$

\section{First-order Digital $\Sigma \Delta$ Modulator (DAC Control) Design}

An accumulator may be considered a simple $1^{\text {st }}$ order $\Sigma \Delta$ modulator as shown in Fig. (4). The 1-bit quantizer outputs is either 0 or 1 when it overflows $[16,18]$. The output of the accumulator, at an arbitrary time is the sum of its input at that time and its contents one clock period earlier. If an overflow occurs, the full scale of the accumulator is subtracted. The output can thus be expressed as [19]:

$$
\begin{aligned}
& \eta_{n}-x_{n}+\eta_{n-1}-y_{t} \\
& \left.y_{t}-x_{n}+\eta_{i-1}-\eta_{i}\right) \\
& \left.y_{i}=x_{i} \quad \Sigma_{i} \quad \Sigma_{-1}\right)
\end{aligned}
$$

The $z$-transformation of eq. (8) is :

$$
y(z)-y(x)-2(z) \cdot\left(1-z^{-1}\right)
$$

The z-transformation of $1^{\text {st }}$ order $\Sigma \Delta$ modulator is:

$$
Y(x)-7^{-\cdot} \cdot x(x)+\left(1-z^{-1}\right) \cdot V(x)
$$

Comparing eq. (9) with eq. (10) shows the similarity between them. Ignoring the latency of one clock period in the signal path of the $\Sigma \Delta$ modulator, and treating the contents of the digital accumulator as the negative of the quantization error, the equations are identical. The design parameters of the $1^{\text {st }}$ order $\Sigma \Delta$ modulator (DAC control) are shown in Table (3 b).

\section{Second-order Digital $\Sigma \Delta$ MASH Modulator (DAC Control) Design}

A second order modulator is constructed by cascading two first-order modulators as shown in Fig. (5). The input is fed to the first stage and the negative of the quantization error from the first stage is used as the input to the second stage. Finally, the outputs of the stage are combined together to form a final output after passing through the error cancellation network, which removes the quantization error components from all the stages except the last one [20]. The output of the second-order MASH modulator is given by eq. (11) [21]. The design parameters of the $2^{\text {nd }}$ order $\Sigma \Delta$ modulator (DAC control) are shown in Table (4).

$Y(z)=F(z)+\left(1-z^{-5}\right)^{2} \cdot E q_{2}(z)$

\section{Third-order Digital $\Sigma \Delta$ MASH Modulator (DAC Control) Design}

A third-order modulator is constructed by cascading three first-order modulators as shown in Fig. (6). The input is fed to the first modulator and the negative of the quantization error from the first and second modulator is used as the input to the second modulator and third modulator, respectively. Finally, the outputs of each modulator are combined together to form a final output after passing through the error cancellation network, which removes the quantization error components from all the modulators except the last one $[16,18]$. The output of the third-order MASH modulator is given by eq. (12) [21]. The design parameters of the $3^{\text {rd }}$ order $\Sigma \Delta$ modulator (DAC control) are shown in Table (5).

$Y(z)=F(z)+\left(--z^{-1}\right) \cdot g_{g}(z)$

\section{Simulation and Results}

The $\mathrm{C}^{++}$language offers the flexibility of computing system behavior in any manner desired. CppSim works in conjunction with Sue2 [22]. CppSim uses Sue2 as its schematic editor for entering designs. Simulations are run through either CppSim or Matlab. The simulation approach taken with CppSim simulator is to represent the various blocks in a system as objects that update their outputs one sample at a time based on inputs that specify one sample at a time. The influences of the inputs on the outputs of each block are determined by their specified behavior, which is set at the beginning of a simulation run. The block behavior can be a function of state information as well as the block inputs. The state information is preserved inside its 
respective block so that the overall simulator does not need to keep tracking it [22]. All blocks of $\Sigma \Delta$ fractional-N PLL synthesizer simulations were done using the CppSim program shown in Fig. (7).

\section{PLL Output Spectrum Simulation}

A $26 \mathrm{MHz}$ reference frequency is used to generate an output frequency of $900 \mathrm{MHz}$. A closed loop bandwidth of $1 \mathrm{MHz}$ is desired with type II PLL. The VCO noise is entered as $-190 \mathrm{dBc} / \mathrm{Hz}$ at $20 \mathrm{MHz}$ offset from the carrier. Phase detector noise, which represents the sum of charge-pump noise, reference jitter and divider jitter is set to $-110 \mathrm{dBc} / \mathrm{Hz}$. The VCO phase noise required for GSM synthesizer is $-162 \mathrm{dBc} / \mathrm{Hz}$ at $20 \mathrm{MHz}$ offset frequency, as shown in Table (1). VCO phase noise to reach $-190 \mathrm{dBc} / \mathrm{Hz}$ at $20 \mathrm{MHz}$ offset frequency is the goal of this paper. In the first simulation of the system, it is assumed that the quantization step size, $\Delta$, of MASH $\Sigma \Delta$ modulator is 1 to show the impact of $\Sigma \Delta$ quantization noise. The simulations for $2^{\text {nd }}, 3^{\text {rd }}$ and $4^{\text {th }}$ order $\Sigma \Delta$ modulator are shown in Figs. (8), (9) and (10) respectively. The simulations show that the GSM phase noise, $i(f)$, specifications are not met in the above design due to the $\Sigma \Delta$ quantization noise which dominates a wide frequency range.

\section{Improvement of phase noise performance for synthesizer}

The overall phase noise performance of the synthesizer, SSB, for $2^{\text {nd }}, 3^{\text {rd }}$ and $4^{\text {th }}$ order $\Sigma \Delta$ modulator is shown in Figs. (11 a), (12 a) and (13 a) respectively. The differences between these simulation results are shown in Table (6). The output spectrum of PFD/DAC PLL synthesizer for $2^{\text {nd }}, 3^{\text {rd }}$ and $4^{\text {th }}$ order $\Sigma \Delta$ modulator is shown in Figs. (11 b), (12 b) and (13 b) respectively.

\section{Discussion of Simulation Results}

1. The quantization noise of $\Sigma \Delta$ modulator is reduced by a factor of $2^{2}$, where $\mathrm{B}$ is the number of bits in the PFD/DAC, so that the quantization noise of $\Sigma \Delta$ modulator becomes below the VCO noise and detector noise.

2. Increasing the order of $\Sigma \Delta$ modulator improves the spurious performance. The quantization noise of the modulator is pushed to high frequencies. To suppress this noise, the loop filter roll off must be at least the same.

3. Mismatch between the unit elements of the PFD/DAC is solved by DWA technique. DWA proves its ability to convert fractional spur noise into a broadband, shaped noise that appears to have the same profile as the quantization noise spectrum.

4. Time mismatch between the two phase paths that generates the charge box is processed by phase swapping. It was found that phase swap improves the spurious performance by converting the spurious energy into a broadband noise source.

5. Sample and hold circuit has improved the performance of the system by eliminating the residual fractional spurs as well as the spur at the reference frequency that occurs due to the mismatch shaping of the PFD/DAC output.

\section{Conclusions}

The $\Sigma \Delta$ fractional-N PLL-FS is designed to generate a frequency signal according to GSM system standard specifications. The simulation results show that the increasing order of the $\Sigma \Delta$ modulator from $2^{\text {nd }}$ order to $4^{\text {th }}$ order improves the phase noise performance of FS by $-186 \mathrm{dBc} / \mathrm{Hz}$ for $3^{\text {rd }}$ order and $-190 \mathrm{dBc} / \mathrm{Hz}$ for $4^{\text {th }}$ order if compared with $2^{\text {nd }}$ order. The minimum numbers of bits required to reduce the effect of quantization noise to the level below the VCO noise are found 14, 13 and 12 bit for $2^{\text {nd }}, 3^{\text {rd }}$ and $4^{\text {th }}$ order $\Sigma \Delta$ modulator respectively. The closed loop bandwidth of PLL is chosen to be $1 \mathrm{MHz}$ to improve the settling time of FS. The settling time results of $2^{\text {nd }}, 3^{\text {rd }}$ and $4^{\text {th }}$ order system are $2.92 \mu \mathrm{s}, 3.28$ $\mu \mathrm{s}$ and $3.38 \mu \mathrm{s}$ respectively. 


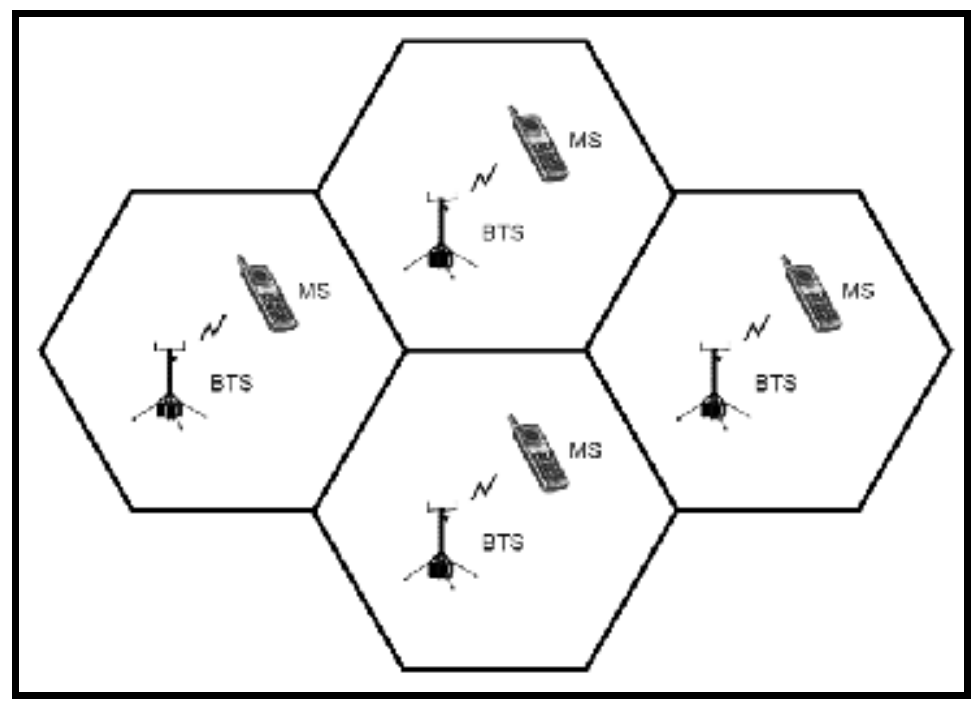

Fig. (1) Cellular system.

Table (1)

Phase noise specifications for GSM 900MHz standard system.

\begin{tabular}{|c||c|}
\hline Offset frequency $(\mathrm{MHz})$ & Phase noise requirement $(\mathrm{dBc} / \mathrm{Hz})$ \\
\hline \hline 3.0 & -123 \\
\hline 6.0 & -129 \\
\hline 10 & -150 \\
\hline
\end{tabular}

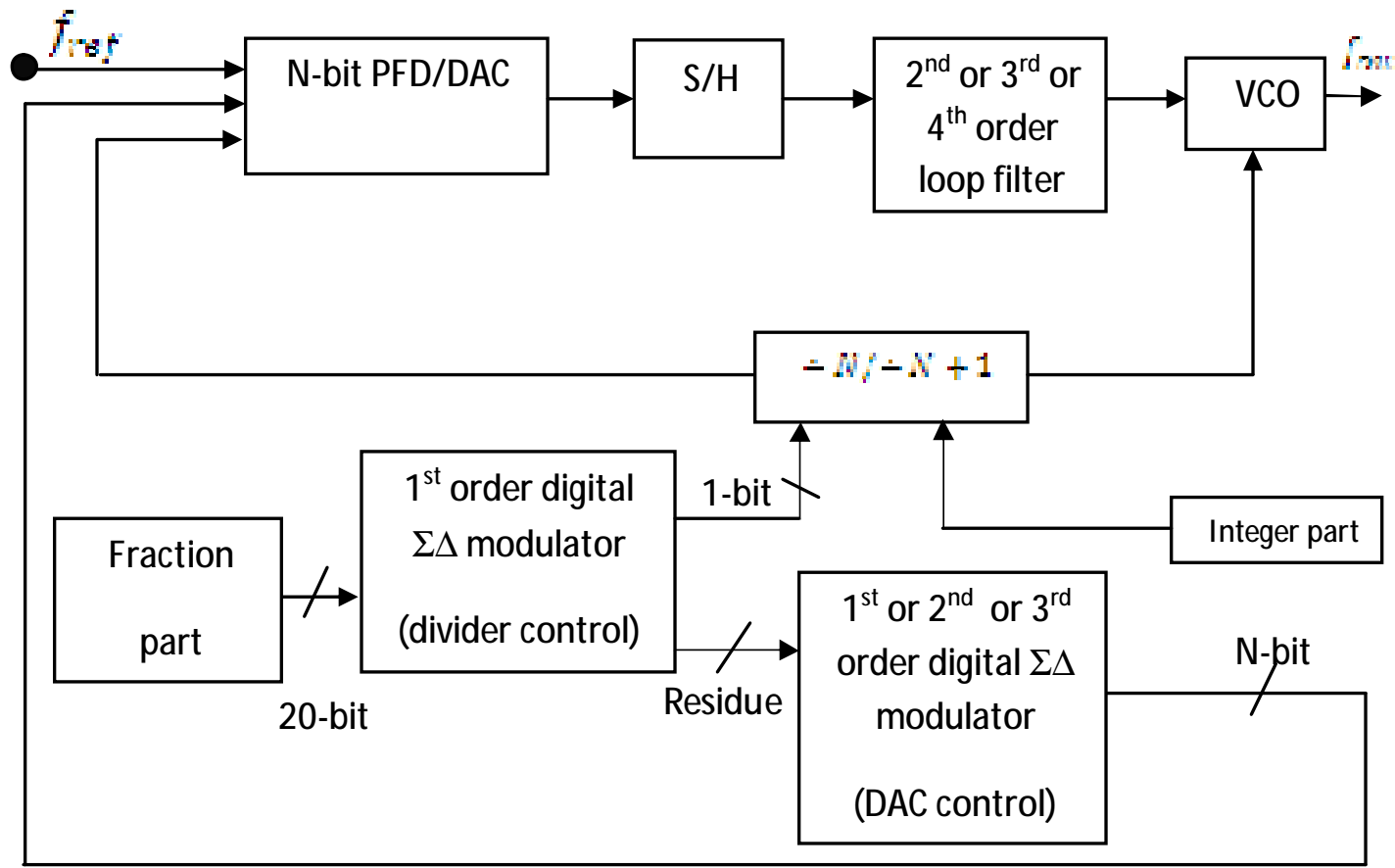

Fig. (2) Block diagram of system. 
Table (2)

Design parameters.

\begin{tabular}{|l|l|}
\hline \multicolumn{1}{|c|}{ Parameter } & \multicolumn{1}{c|}{ Proposed system parameters } \\
\hline \hline Output frequency & $900 \mathrm{MHz}$ \\
\hline Reference frequency & $26 \mathrm{MHz}[5]$ \\
\hline Loop bandwidth & $1 \mathrm{MHz}$, To improve settling time \\
\hline Charge pump current & $5 \mathrm{~mA}$ \\
\hline Filter (Order, Type) & $2^{\text {nd }}, 3^{\text {rd }}$ and $4^{\text {th }}$ order, passive type \\
\hline$\Sigma \Delta$ modulator (Order, Type) & $2^{\text {nd }}, 3^{\text {rd }}$ and $4^{\text {th }}$ order, MASH type \\
\hline Divider value & 34.6154 \\
\hline PFD/DAC resolution & $14-$-bit $\left(2^{\text {nd }}\right.$ order $), 13-b i t \quad 3^{\text {rd }}$ order $)$ and $12-b i t$ \\
$\left(4^{\text {th }}\right.$ order $)$
\end{tabular}

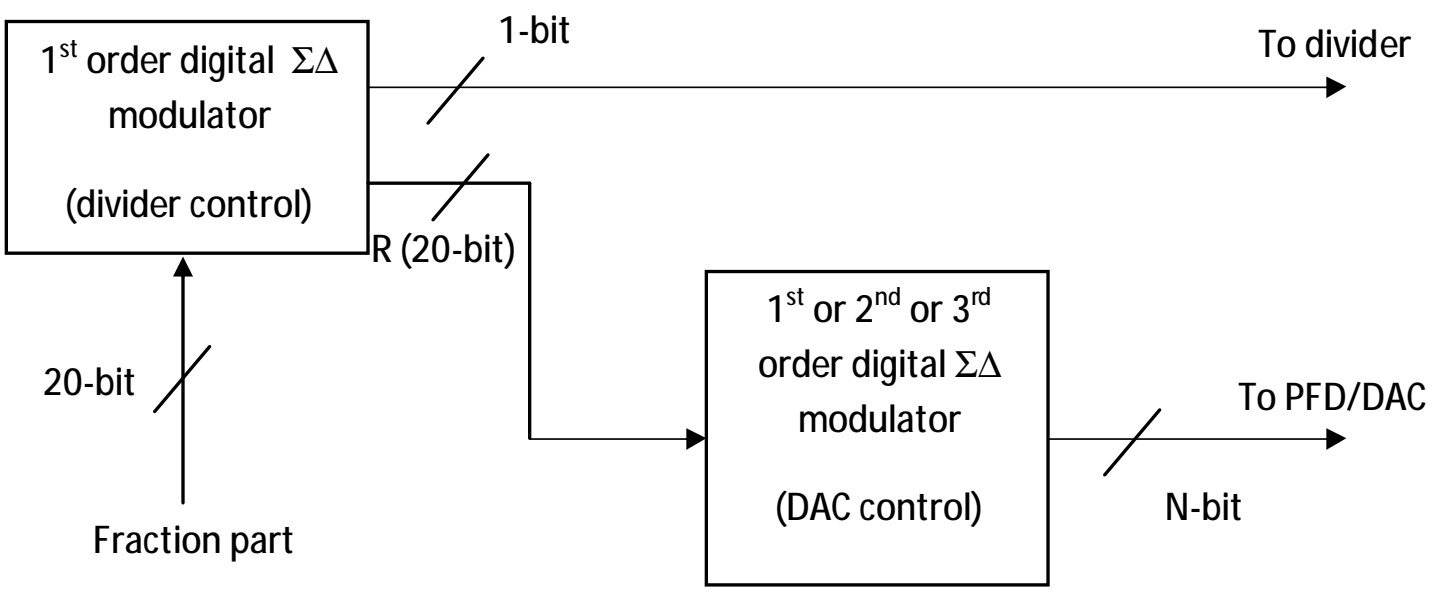

Fig. (3) Model of noise shaped in PFD/DAC. 

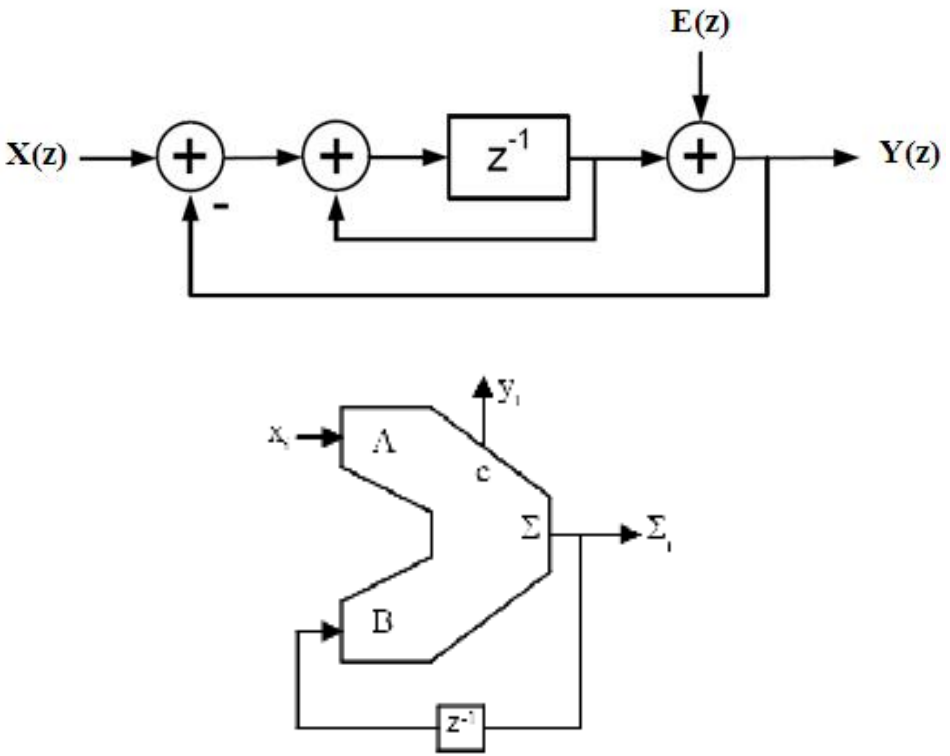

Fig. (4) Similarity of the $\Sigma \Delta$ modulator and the accumulator.

Table (3 a)

$1^{\text {st }}$ order $\Sigma \Delta$ modulator (divider control) parameters.

\begin{tabular}{|c|c|}
\hline Parameters & Value \\
\hline \hline Input bit & 20-bits \\
\hline \hline Output bit & 1-bits \\
\hline Fractional resolution & $25 \mathrm{~Hz}$ \\
\hline Sampling frequency & $26 \mathrm{MHz}$ \\
\hline
\end{tabular}

Table (3 b)

$I^{\text {st }}$ order $\Sigma \Delta$ modulator (DAC control) parameters.

\begin{tabular}{|c||c|}
\hline Parameters & Value \\
\hline \hline Input bit & 20-bits \\
\hline Output bit & 14-bits \\
\hline Fractional resolution & $25 \mathrm{~Hz}$ \\
\hline Sampling frequency & $26 \mathrm{MHz}$ \\
\hline
\end{tabular}

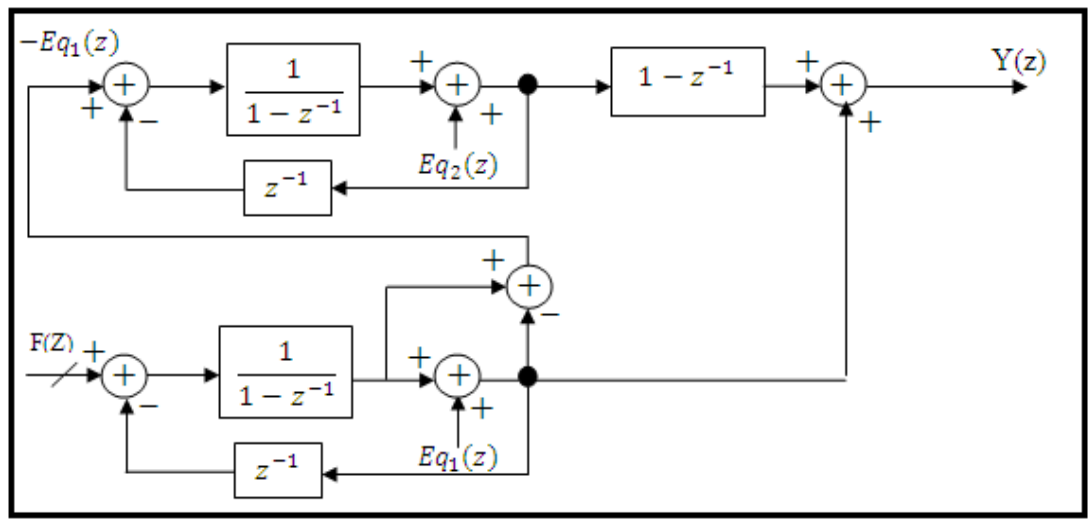

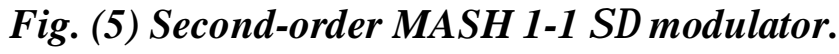


Table (4)

$2^{\text {nd }}$ order $\Sigma \Delta$ modulator parameters.

\begin{tabular}{|c||c|}
\hline \multicolumn{1}{|c||}{ Parameters } & Value \\
\hline \hline Input bit & 20 -bits \\
\hline Output bit & 13 -bits \\
\hline Fractional resolution & $25 \mathrm{~Hz}$ \\
\hline Sampling frequency & $26 \mathrm{MHz}$ \\
\hline
\end{tabular}

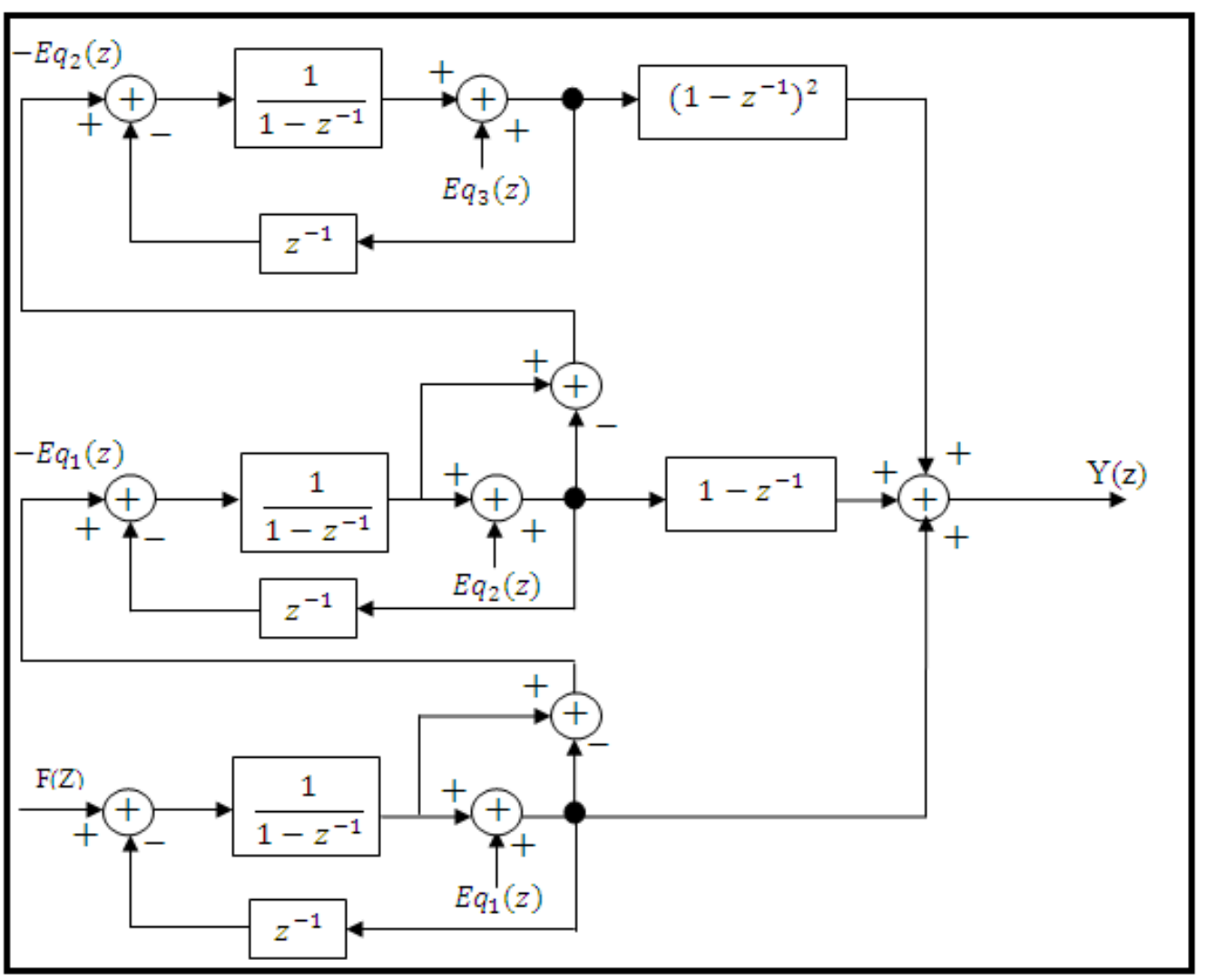

Fig. (6) Third-order MASH 1-1-1 $\Sigma \Delta$ modulator.

Table (5)

$3^{\text {rd }}$ order $\Sigma \Delta$ modulator parameters.

\begin{tabular}{|c|c|}
\hline Parameters & Value \\
\hline Input bit & 20-bits \\
\hline Output bit & $12-$ bits \\
\hline Fractional resolution & $25 \mathrm{~Hz}$ \\
\hline Sampling frequency & $26 \mathrm{MHz}$ \\
\hline
\end{tabular}




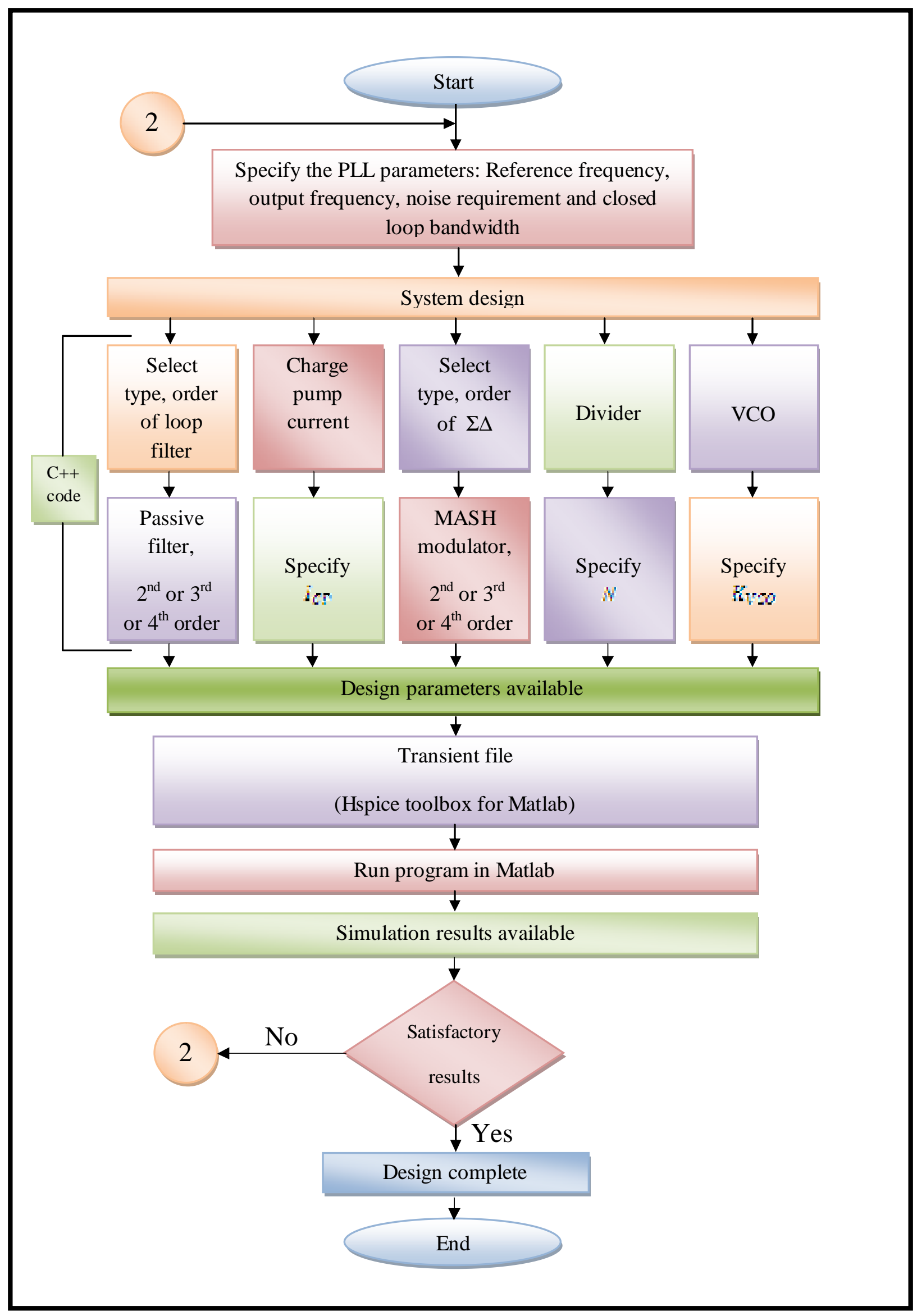

Fig. (7) Flow chart of the $\Sigma \Delta$ fractional-N PLL simulation program. 


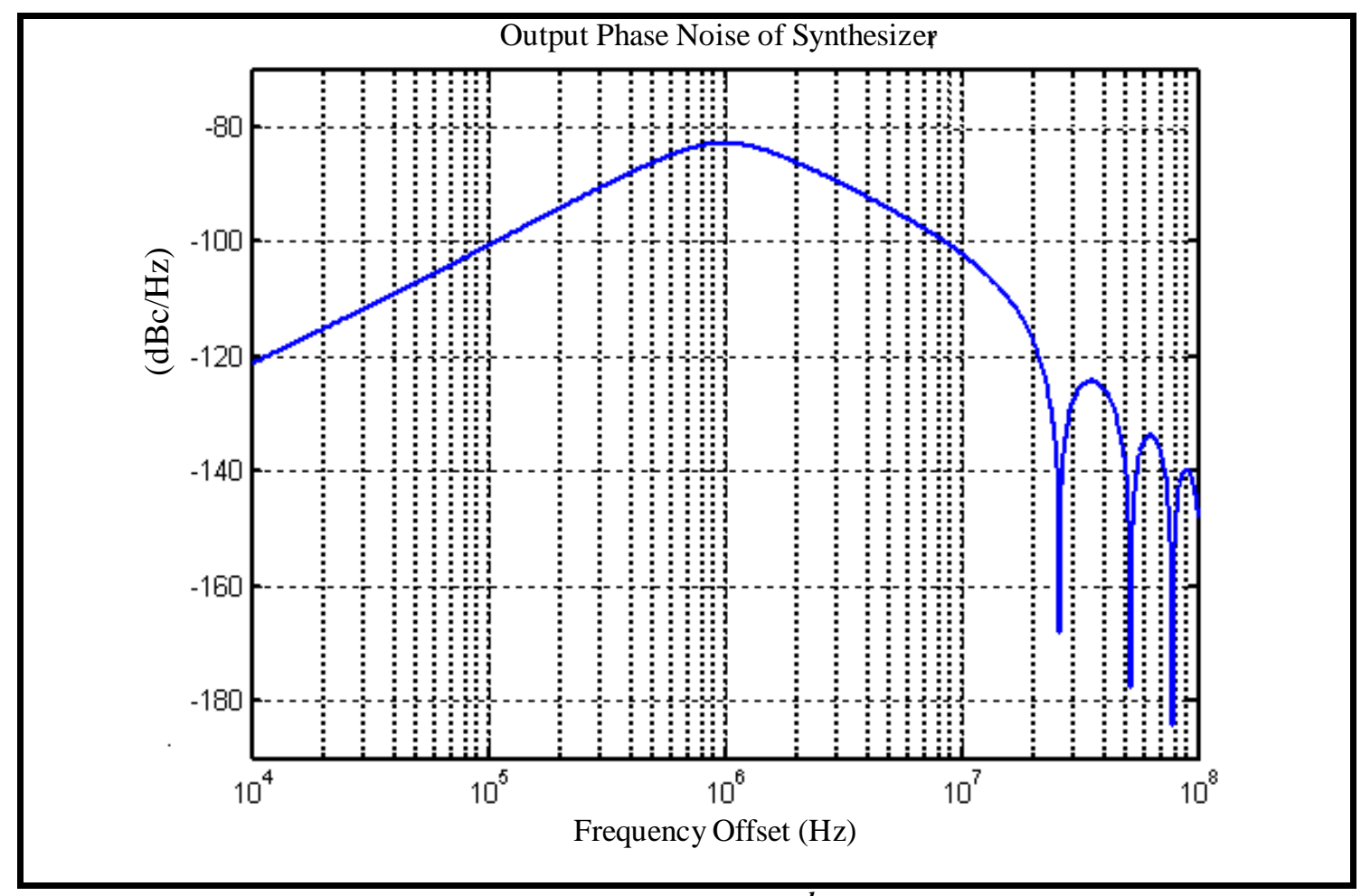

Fig. (8) Simulation showing the impact of $2^{\text {nd }}$ order $\Sigma \Delta$ quantization noise.

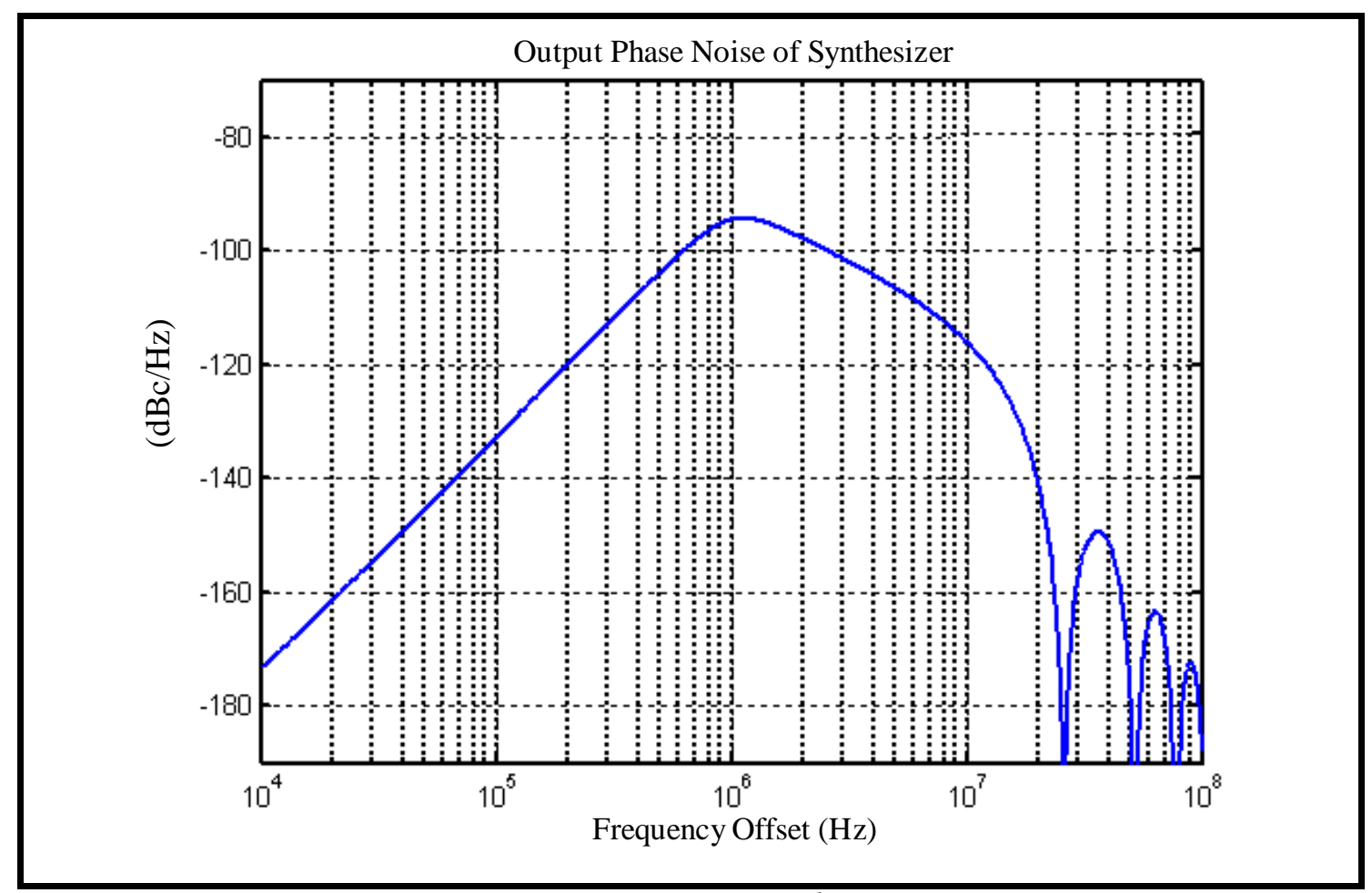

Fig. (9) Simulation showing the impact of $3^{\text {rd }}$ order $\Sigma \Delta$ quantization noise. 


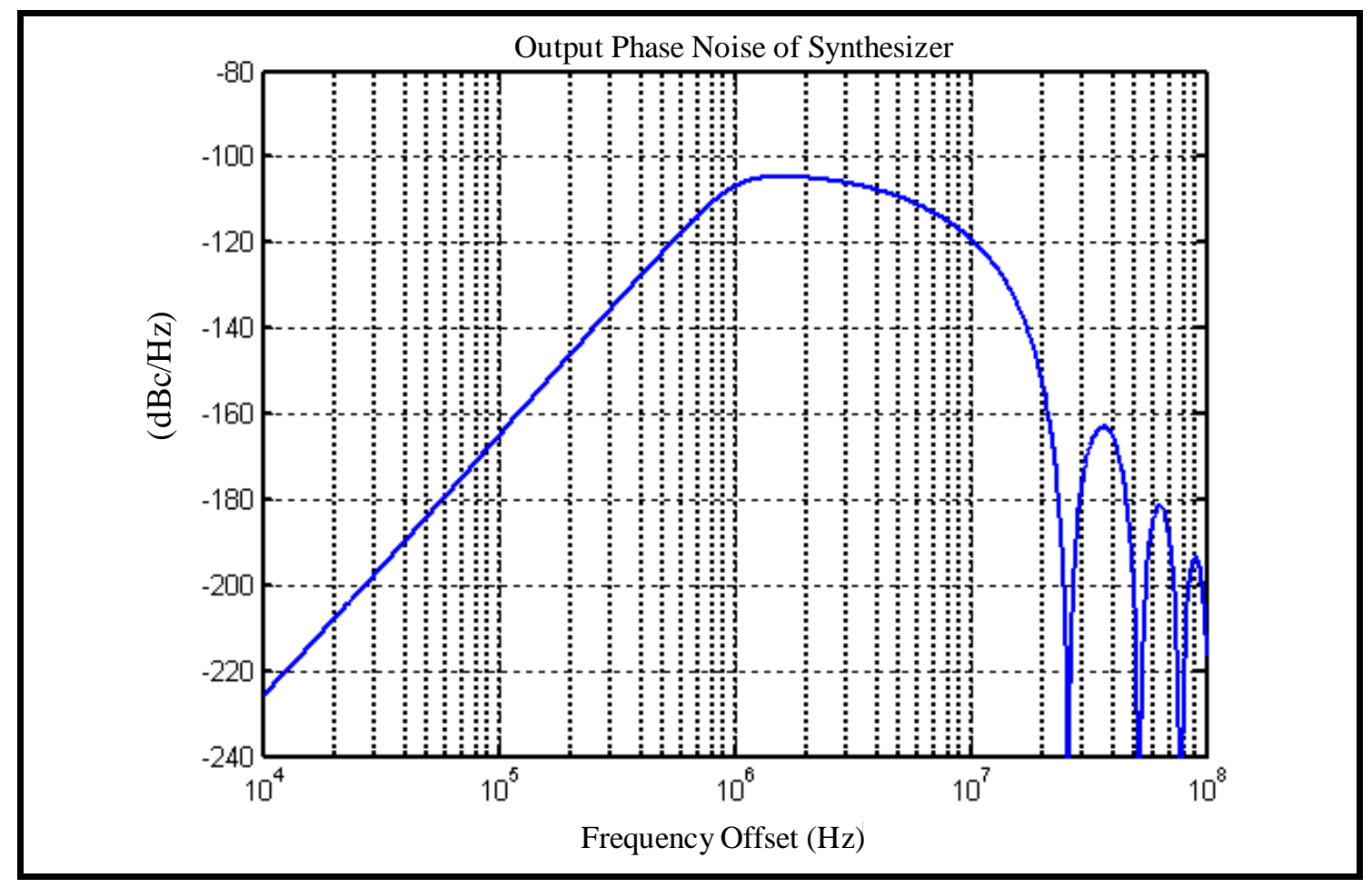

Fig. (10) Simulation showing the impact of $4^{\text {th }}$ order $\Sigma \Delta$ quantization noise.

Table (6)

Phase noise simulation results.

\begin{tabular}{||c||c||c|c|c||}
\hline $\begin{array}{c}\text { Offset } \\
\text { frequency }\end{array}$ & $\begin{array}{c}\text { Phase noise } \\
\text { requirement } \\
(\mathrm{dBc} / \mathrm{Hz})\end{array}$ & $\begin{array}{c}\text { Phase noise result } \\
\text { for } 2^{\text {nd }} \text { order } \\
\text { system }(\mathrm{dBc/Hz})\end{array}$ & $\begin{array}{c}\text { Phase noise result } \\
\text { for } 3^{\text {rd }} \text { order } \\
\text { system }(\mathrm{dBc} / \mathrm{Hz})\end{array}$ & $\begin{array}{c}\text { Phase noise result } \\
\text { for } 4^{\text {th }} \text { order } \\
\text { system }(\mathrm{dBc} / \mathrm{Hz})\end{array}$ \\
\hline \hline $3.0 \mathrm{MHz}$ & -123 & -134 & -141 & -142 \\
\hline $6.0 \mathrm{MHz}$ & -129 & -144 & -159 & -162 \\
\hline $10 \mathrm{MHz}$ & -150 & -154 & -171 & -180 \\
\hline \hline $20 \mathrm{MHz}$ & -162 & -164 & -186 & -190 \\
\hline
\end{tabular}




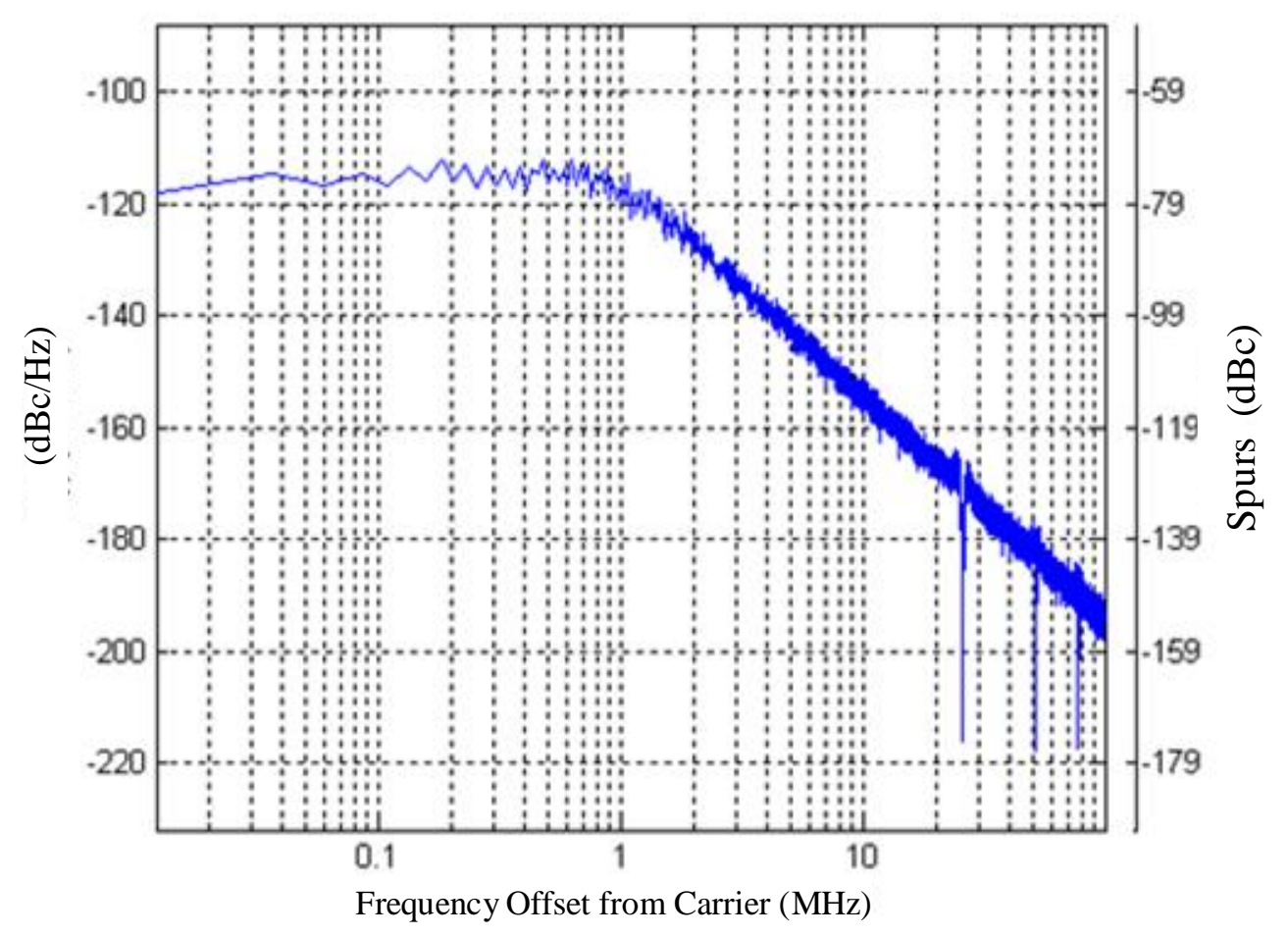

Fig. (11 a) Simulation showing the overall phase noise performance for $2^{\text {nd }}$ order $\Sigma \Delta$ modulator.

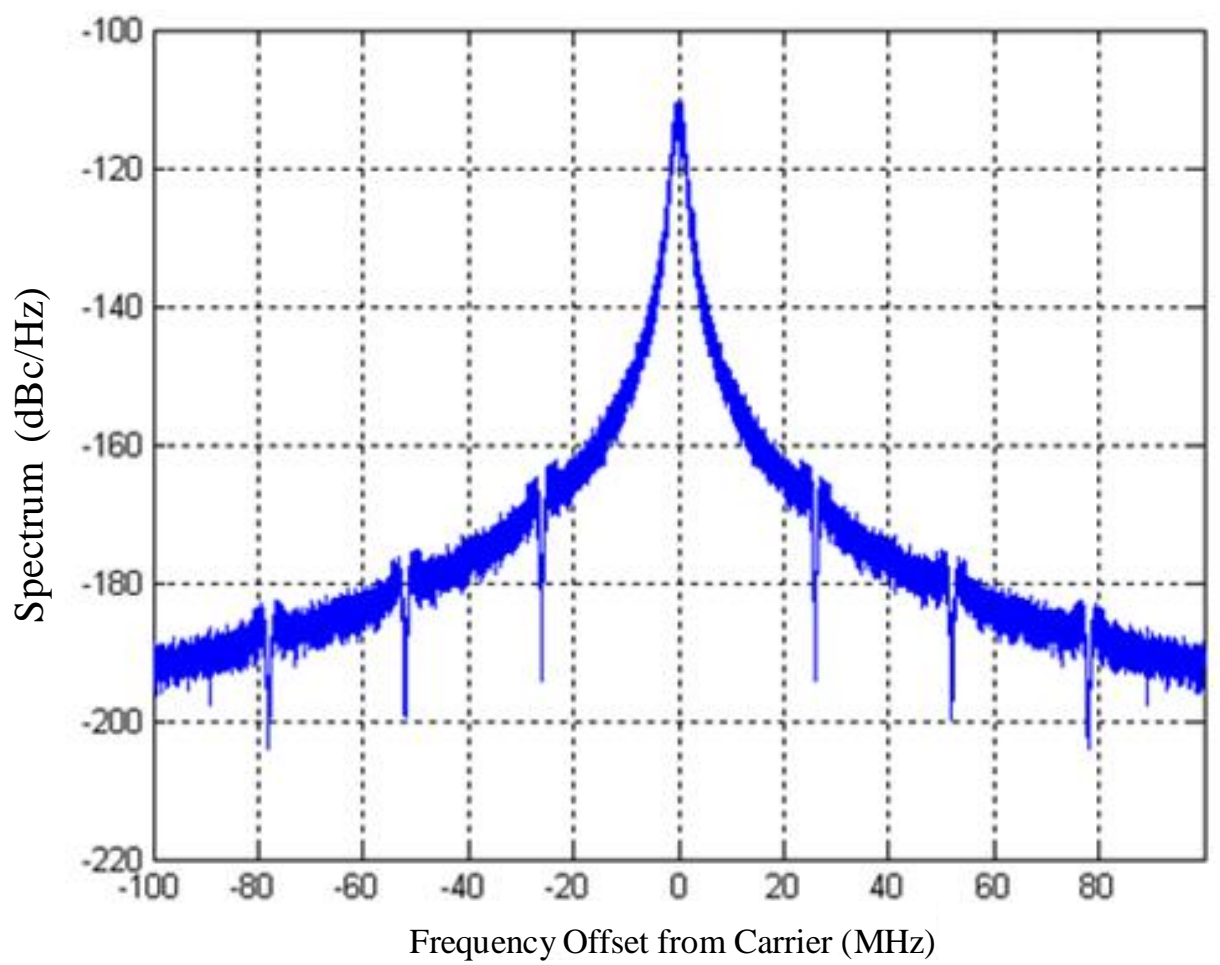

Fig. (11 b) Simulation showing the output spectrum of PLL synthesizer for $2^{\text {nd }}$ order $\Sigma \Delta$ modulator. 


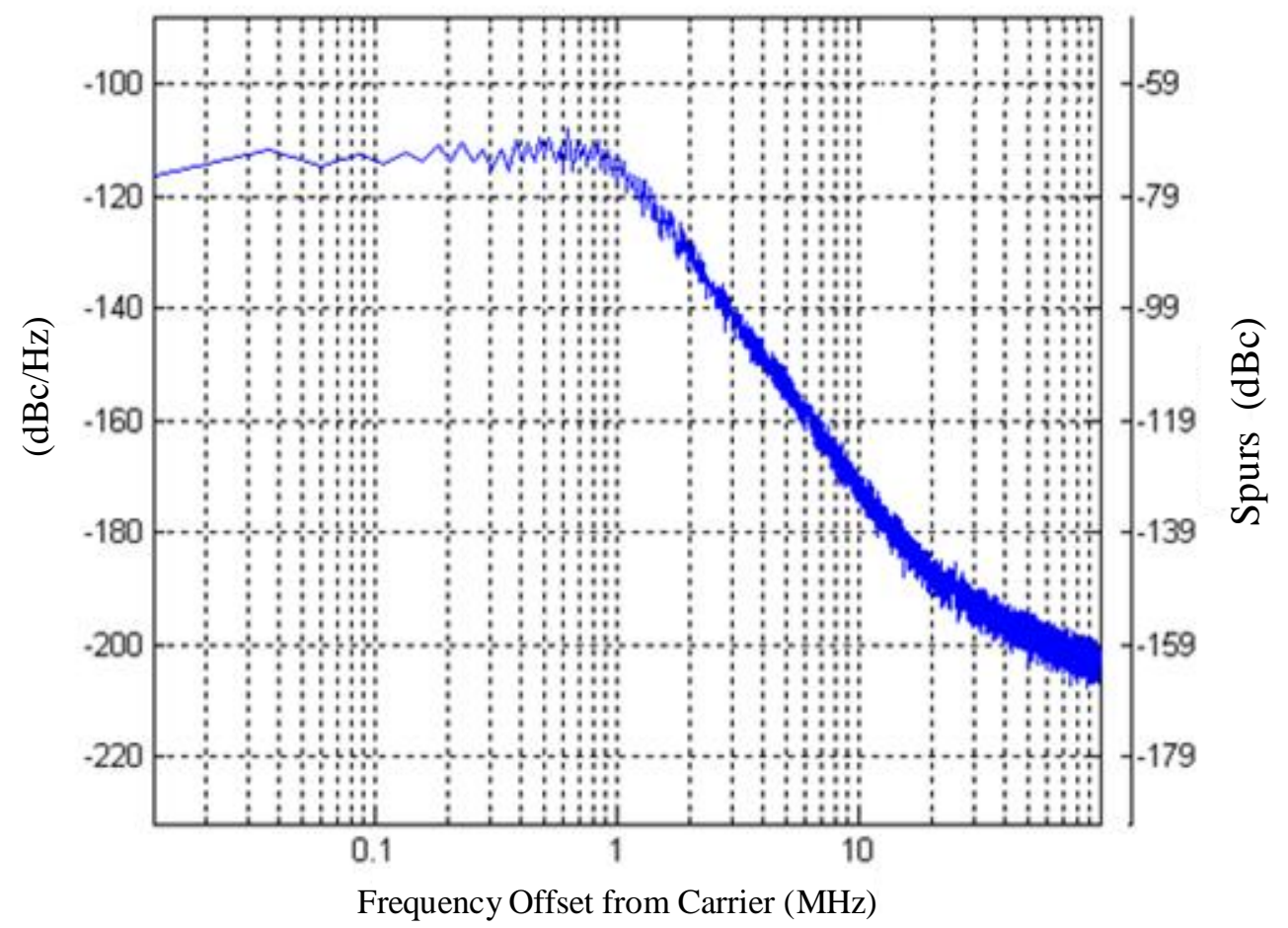

Fig. (12 a) Simulation showing the overall phase noise performance for $3^{\text {rd }}$ order $\Sigma \Delta$ modulator.

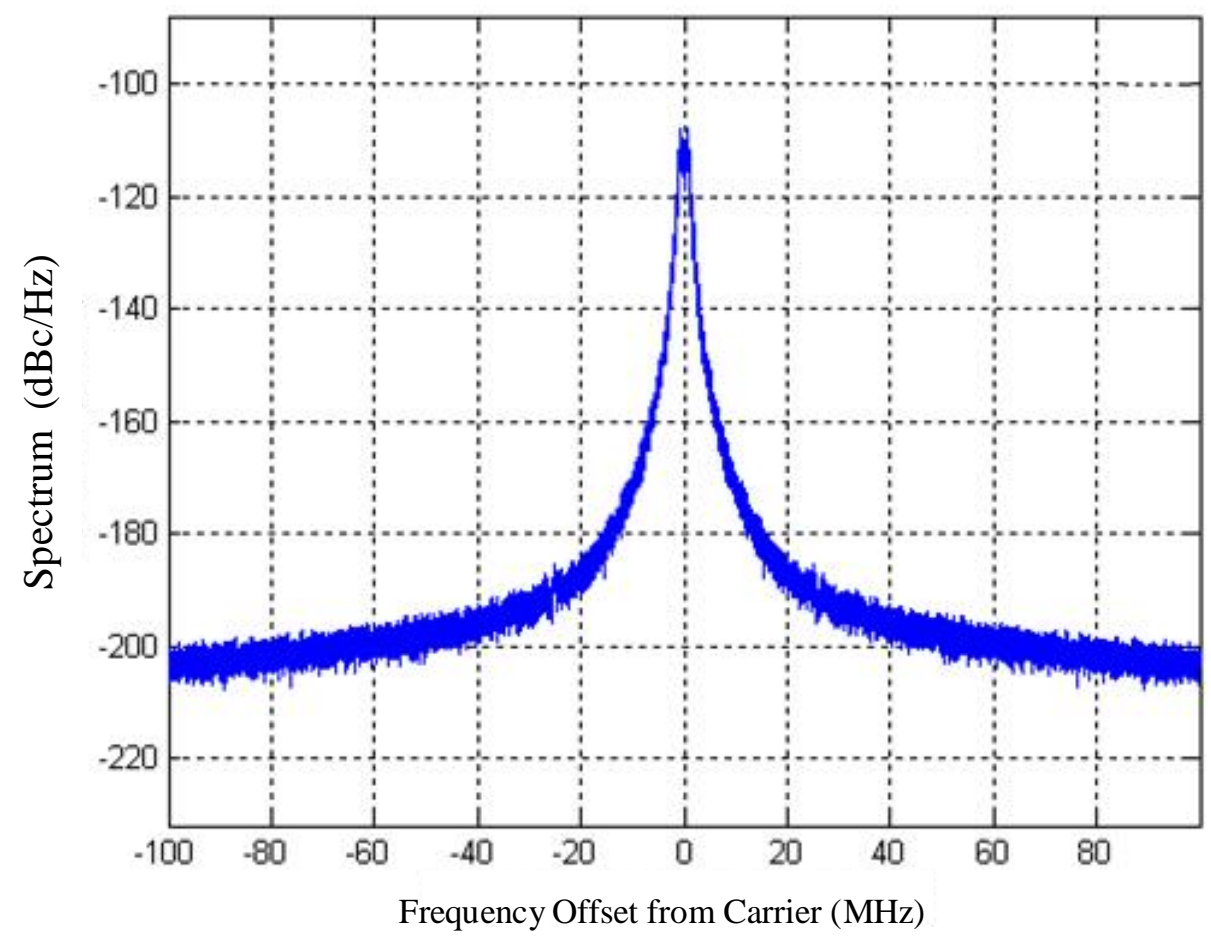

Fig. (12 b) Simulation showing the output spectrum of PLL synthesizer for $3^{\text {rd }}$ order $\Sigma \Delta$ modulator. 


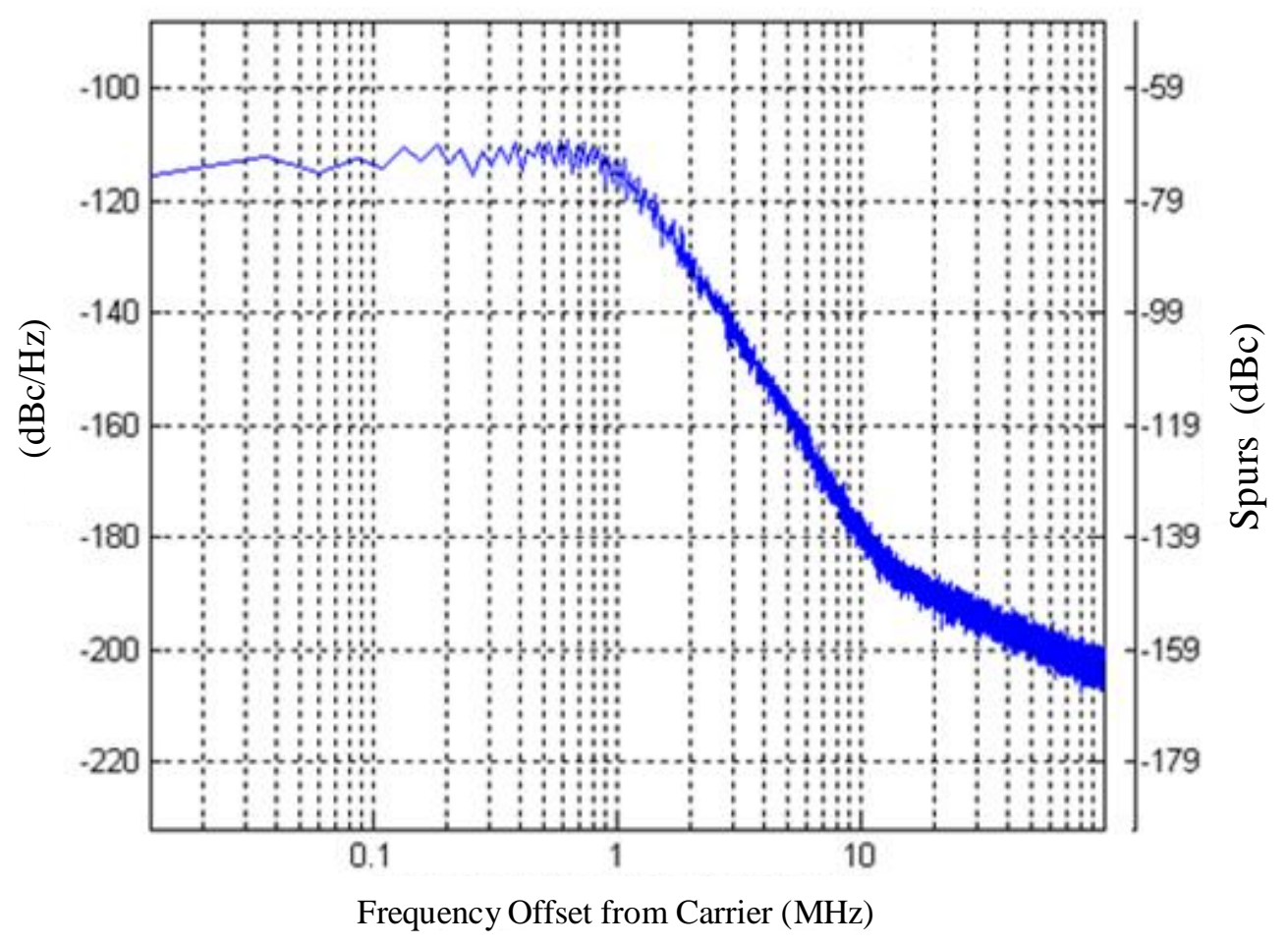

Fig. (13 a) Simulation showing the overall phase noise performance for $4^{\text {th }}$ order $\Sigma \Delta$ modulator.

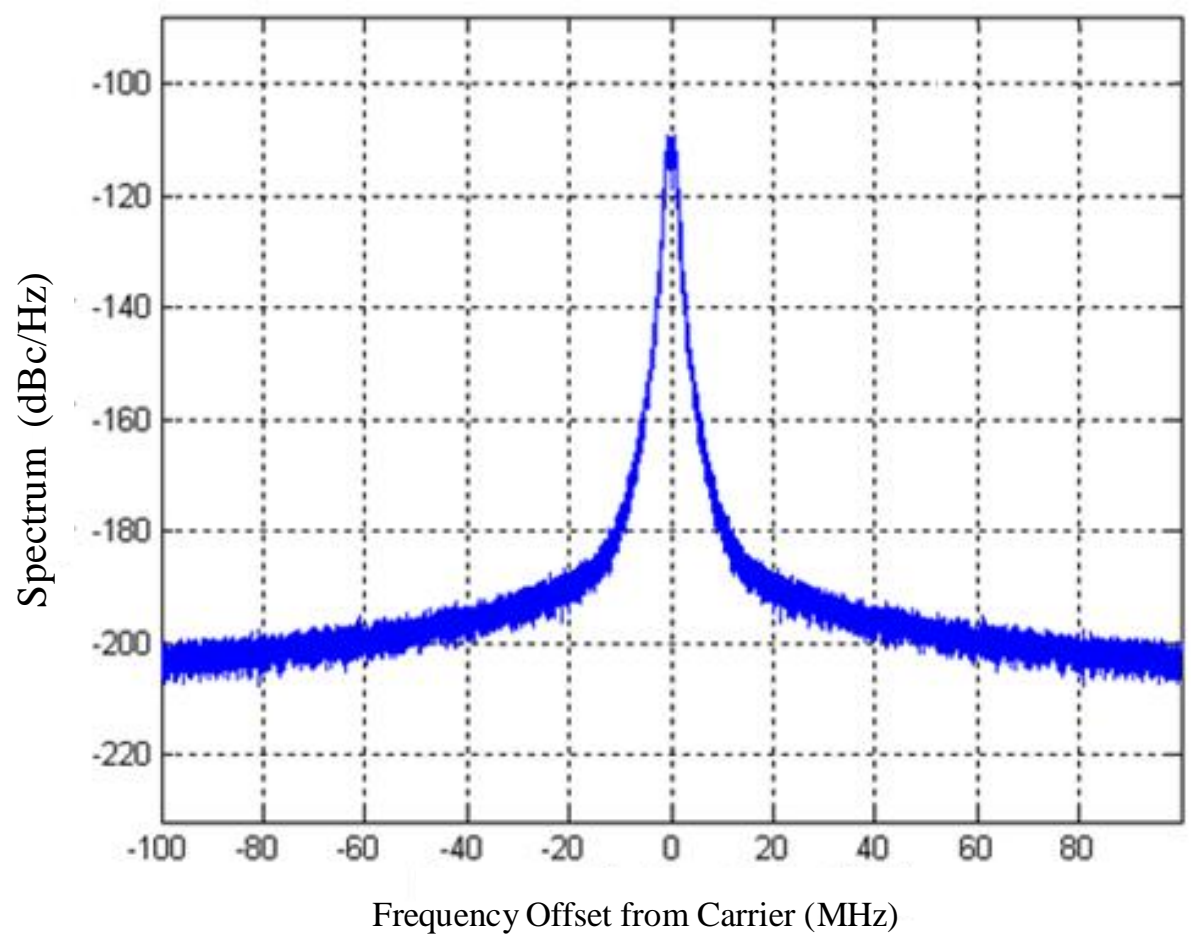

Fig. (13 b) Simulation showing the output spectrum of PLL synthesizer for $4^{\text {th }}$ order $\Sigma \Delta$ modulator. 


\section{References}

[1] B. DeMuer \& M. Steyaert, "CMOS Fractional-N Synthesizers: Design for High Purity and Monolithic Integration", Kluwer Academic Publishers, 2003.

[2] J. Vankka, "Digital Synthesizers and Transmitters for Software Radio", Springer, 2005.

[3] A. V. Roermund, M. Steyaert \& J. H. Huijsing, "Analog Circuit Design : Fractional-N Synthesizers, Design for Robustness, Line and Bus Drivers", Kluwer Academic Publishers, 2003.

[4] H. Xiuyan, "GSM Fundamentals", Huawei Technologies Co., Ltd, 2006, http//www. huawei.com.

[5] J. Crols \& M. Steyaert, "CMOS Wireless Transceiver Design", Kluwer Academic Publishers, 1997.

[6] B. G. Goldberg, "Digital Frequency Synthesis Demystified: DDS and Fractional-N PLLs", LLH Technology Publishing, 1999.

[7] C. Barrett, "Fractional/Integer-N PLL Basics", Texas Instruments, Wireless Communication Business Unit, Technical Brief SWRA029, August 1999.

[8] C. S. Vaucher, "Architectures for RF Frequency Synthesizers", Kluwer Academic Publishers, 2003.

[9] V. Manassewitsch, "Frequency Synthesizers: Theory and Design", Third edition, John Wiley \& Sons, Inc., 1987.

[10] A. Fox, "PLL Synthesizers", Analog Devices, 2002, http//www. analog. com/library.

[11] S. E. Meninger, "Design of a Wideband Fractional-N Frequency Synthesizer Using CppSim", May 2005, www. mtl. mit. edu/research-groups/ perrottgroup/ tools.html.

[12] S. E. Meninger, "Low Phase Noise, High Bandwidth Frequency Synthesis Techniques", Ph.D. Thesis, Massachusetts Institute of Technology, Cambridge, May 2005.

[13] S. E. Meninger \& M. H. Perrott, "A Fractional-N Frequency Synthesizer Architecture Utilizing a Mismatch Compensated PFD/DAC Structure for
Reduced Quantization-Induced Phase Noise", IEEE Transactions on Circuits and Systems-II: Analog and Digital Signal Processing, Vol. 50, No. 11, pp. 839-849, November 2003.

[14] M. Kozak \& E. G. Friedman, "Design and Simulation of Fractional-N Frequency Synthesizers", IEEE, ISCAS, pp. 780783, 2004.

[15] H. M. Azawi, "Design and Performance Evaluation of Sigma-Delta Modulator Fractional-N Frequency Synthesizer", M. Sc. Thesis, Department of Electrical and Electronic Engineering, University of Technology, Baghdad, 2007.

[16] A. Howard, "Delta-Sigma Modulator PLLs With Dithered Divide Ratio", Agilent Corp., 2001, http//www. agilent. com.

[17] D. Jarman, "A Brief Introduction to Sigma Delta Conversion", Intersil Corp., Application Note 9504, May 1999.

[18] S. H. Kim, M. S. Keel, K. Lee \& S. Kim, "CMOS Delta-Sigma Frequency Synthesizer with a New Frequency Divider and a Simplified MASH Structure", Journal of the Korean Physical Society, Vol. 41, No. 6, pp. 967973, December 2002.

[19] R. Ahola, "Integrated Radio Frequency Synthesizers for Wireless Applications", $\mathrm{Ph}$. D. Thesis, Helsinki University of Technology, April 2005.

[20] M. Kozak \& I. Kale, "Oversampled Delta-Sigma Modulators: Analysis, Applications and Novel Topologies", Kluwer Academic Publishers, 2003.

[21] S. Park, "Principles of Sigma-Delta Modulation for Analog-to-Digital Converters", Motorola, Inc., Application Note APR8, 1997.

[22] M. H. Perrott, "CppSim Reference Manual”, 2002, http://www-mtl. mit. edu/ perrott. 


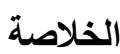

في هذا البحث تم مناقثة, تحليل, تصميم وتقييم المحاكاة

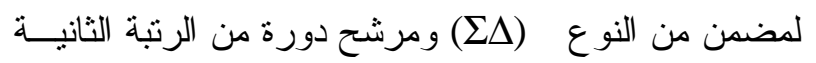

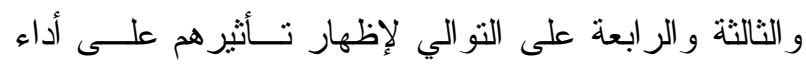

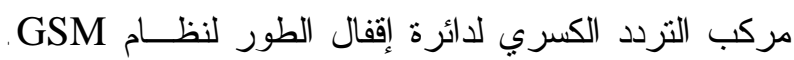

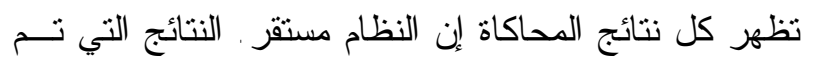

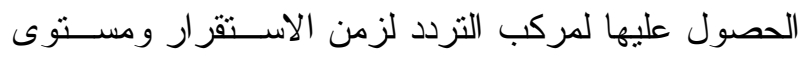
التردد الطيفي وضوضـاء الطور عنـــ (20 MHz offset)

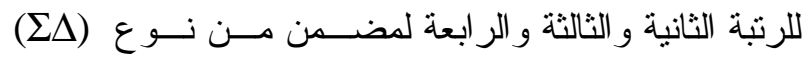

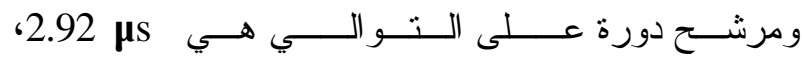
- 6-64 dBc ،3.28 $\mu \mathrm{s}$ ، $-164 \mathrm{dBc} / \mathrm{Hz}$ ، $-35 \mathrm{dBc}$ $-190 \mathrm{dBc} / \mathrm{Hz}$ g-79 dBc ،3.38 $\mu \mathrm{s}$ ، $186 \mathrm{dBc} / \mathrm{Hz}$

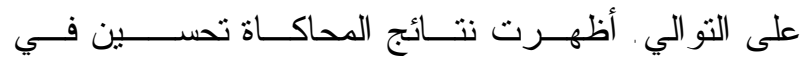

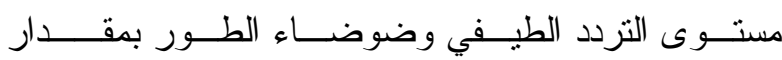
31 dBc/Hz،-19 Bc 35 dBc/Hz

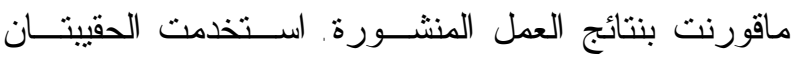
البرمجيتان Matlab (R2007a) CppSim في محاكاة

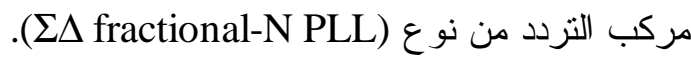

